\title{
Design and development of high-frequency thermoacoustic engines for thermal management in microelectronics
}

\author{
O.G. Symko*, E. Abdel-Rahman, Y.S. Kwon, M. Emmi, R. Behunin \\ Department of Physics, University of Utah, 115 S. 1400 E., Room 201, Salt Lake City, UH 84112-0830, USA
}

Received 15 November 2002; revised 28 August 2003; accepted 24 September 2003

\begin{abstract}
Thermoacoustic heat engines provide a practical solution to the problem of heat management in microcircuits where they can be used to pump heat or produce spot cooling of specific circuit elements. There are basically two types of thermoacoustic engines, a prime mover where heat is converted to acoustic energy, and a heat pump or cooler where sound can pump heat up a temperature gradient. Such devices are relatively simple, they can be efficient, and they are readily adaptable to microcircuit interfacing. Since this type of engines is usually operated in a resonant mode, the operating frequency determines its size. The devices presented here are pumped at frequencies ranging from 4 to $24 \mathrm{kHz}$. They have been developed for interfacing with microcircuits as heat pumps or spot coolers. Results of their performance are presented and suggestions for further improvements are discussed.

(C) 2003 Elsevier Ltd. All rights reserved.
\end{abstract}

Keywords: Heat management; Thermoacoustic; Miniaturization; Acoustic heat pump; Engine

\section{Introduction}

The need to manage heat fluxes of order $10-50 \mathrm{~W} / \mathrm{cm}^{2}$ and higher in microcircuits has emphasized the importance of developing devices which can cope with such heat levels. Many interesting devices have been proposed for such applications. They range from forced convection cooling devices to thermoelectric devices, heat pipes, liquid coolants, and evaporative spray cooling devices [1]. Here thermoacoustic engines are proposed; such engines can convert heat to sound or use sound to pump heat. They are a new application in the area of thermal management but based on their performance and adaptability to microcircuits they show much promise [2,3].

A thermoacoustic heat engine consists of a working gas, in the present case it is air at one atmosphere, a medium for thermal rectification called the stack, heat exchangers at each end of the stack, an acoustic resonator, and a loudspeaker when the engine is a heat pump or a temperature difference when it is used as a converter of

\footnotetext{
* Corresponding author. Tel.: + 1-801-581-6132; fax: + 1-801-581-4801. E-mail addresses: orest@physics.utah.edu (O.G. Symko), ehab@ physics.utah.edu (E. Abdel-Rahman), y.s.kwon@physics.utah.edu (Y.S. Kwon), matthew@audiophile.com (M. Emmi).
}

heat to sound (known as prime mover). In many of the large scale applications of thermoacoustic engines, discussed by Swift [4] and Garrett and Hofler [5], a typical operating frequency has been around $500 \mathrm{~Hz}$. Since the devices are usually pumped in the resonant mode with mainly standing wave components, it is possible to scale down their dimensions by raising the acoustic frequency. The devices presented here have been scaled down in size and hence they operate at much higher frequencies, in the range of 4-24 kHz; they demonstrate how acoustic devices can be made small enough for interfacing with microcircuits and how they are being developed for such applications.

The prime mover type of engine has a long history, going back to the eighteenth century. Rayleigh explained the basic phenomenon as an example of a relaxation oscillator [6]. Acoustic Refrigeration was first demonstrated in 1975 when a section of a resonance tube showed cooling when acoustically driven [7].

Theoretical aspects of thermoacoustics have been covered in a series of papers by Rott [8], and Rott and Zouzoulas $[9,10]$ with linearization of Navier-Stokes equation; they were related to experimental results by Swift [11]. Following this an acoustic refrigerator was developed by Wheatley, Hofler, Swift, and Migliori [12]. Based on the above thermoacoustic phenomena, the total 


\begin{tabular}{|c|c|c|c|}
\hline \multicolumn{2}{|c|}{ Nomenclature } & $\delta_{\kappa}$ & thermal penetration depth \\
\hline$A$ & cross-sectional area & $\delta_{\mathrm{v}}$ & viscous penetration depth \\
\hline $\begin{array}{l}A \\
c_{n}\end{array}$ & specific heat at constant pressure & $\Pi$ & total effective circumference around stack, per- \\
\hline K & thermal conductivity & & pendicular to heat flow in stack \\
\hline$p_{1}$ & amplitude of acoustic pressure change of sound & $\Gamma$ & $\begin{array}{l}\text { ratio of temperature gradient across stack to } \\
\text { critical temperature gradient }\end{array}$ \\
\hline$\dot{Q}_{2}$ & rate of heat flux & $\rho_{m}$ & density of working fluid \\
\hline$u_{1} \mathrm{~s}$ & particle velocity in sound wave & $\omega$ & acoustic radian frequency \\
\hline$T$ & $\begin{array}{l}\text { temperature } \\
\text { teme }\end{array}$ & $\eta$ & thermal efficiency, which is the work achieved \\
\hline$\Delta x$ & length of stack in direction of acoustic field & & \\
\hline$\beta$ & thermal expansion coefficient & & \\
\hline
\end{tabular}

rate of heat flux $\dot{Q}_{2}$ pumped acoustically along the stack is given approximately (when viscous losses are neglected) by

$\dot{Q}_{2}=-\frac{1}{4} \Pi \delta_{\kappa} T_{m} \beta p_{1} u_{1}(\Gamma-1)$

where $\Pi \delta_{\kappa}$ is the total effective area perpendicular to the heat flow direction along the stack material, $\delta_{\kappa}$ is the thermal penetration depth next to each element of the stack representing average distance over which sound field interacts thermally with a stack element, $\mathrm{T}_{m} \beta$ are working gas parameters with $\beta$ being the thermal expansion coefficient of the gas and $\mathrm{T}_{m}$ its mean absolute temperature, $p_{1} u_{1}$ represents the acoustic intensity inside the resonator with $p_{1}$ being the amplitude pressure oscillations and $u_{1}$ the amplitude particle velocity; $\Gamma$ is the ratio of the temperature gradient across the stack $\nabla \mathrm{T}_{m}$ to a critical temperature gradient $\nabla T_{\text {crit }}$, which depends on geometry, gas properties and acoustic drive. Fig. 1 shows the thermal penetration depth around a fiber of the stack. The derivation of Eq. (1) is discussed by Rott [10] and Swift [11]. When $\nabla \mathrm{T}_{m} \gg \nabla \mathrm{T}_{\text {crit }}$ heat flux is toward the nearest pressure node in the resonator (as in a prime mover) while for $\nabla \mathrm{T}_{m}<\nabla \mathrm{T}_{\text {crit }}$ heat flux is away from the pressure node as in a refrigerator. Eq. (1) is based on the change of entropy of the gas next to the stack and its oscillations within the stack in the presence of a sound field. The total rate of acoustic power absorbed or produced by the stack is given approximately by (again neglecting viscous losses)

$\dot{W}_{2}=\frac{1}{4} \Pi \delta_{\kappa} \Delta x \frac{T_{m} \beta^{2} \omega}{\rho_{m} c_{p}} p_{1}{ }^{2}(\Gamma-1)$

where $\Delta x$ is the length of stack in the direction of the acoustic field, $\omega$ the acoustic angular frequency, $\rho_{m}$ the gas density and $c_{p}$ its specific heat. This equation comes from the work flow which is the work per cycle times the rate at which this work occurs $[10,11]$.

Here as well the magnitude of the ratio of temperature gradients $\Gamma$ determines whether the engine is a prime mover or a heat pump, where heat can produce sound or sound can pump heat, respectively. Combining Eqs. (1) and (2), one can evaluate the theoretical performance of the two types of engines. The thermal efficiency of the prime mover can then be written in a form which relates to a Carnot efficiency of an engine at $T_{m}$

$\eta=\left(\frac{\Delta T}{T_{m}}\right) \frac{1}{\Gamma}$

while the Coefficient of Performance, the C.O.P. of the refrigerator is also related to a device at $T_{m}$

C.O.P. $=\left(\frac{T_{m}}{\Delta \mathrm{T}}\right) \Gamma$

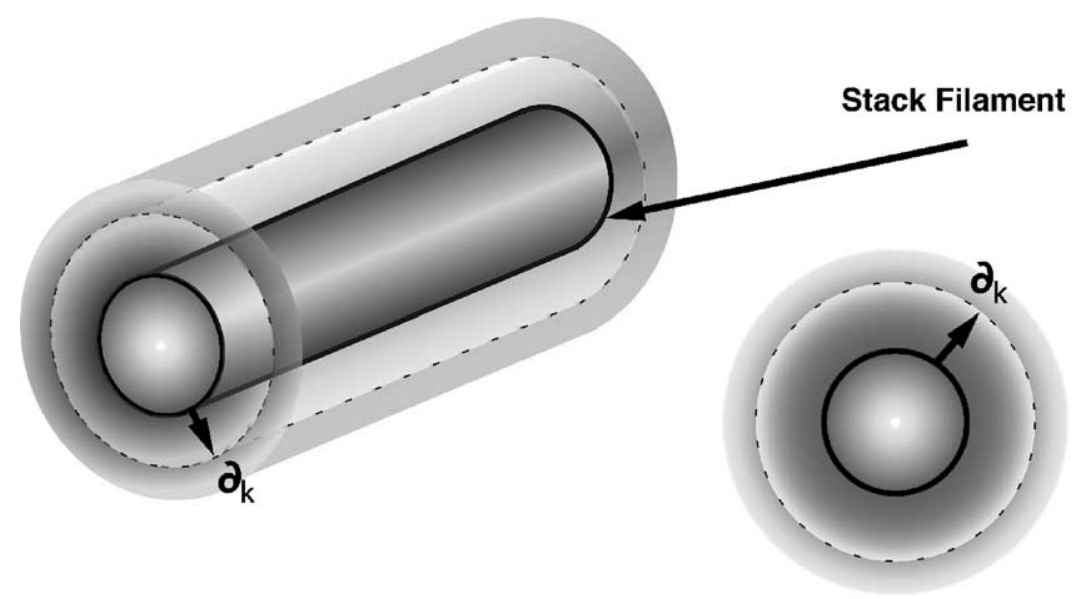

Fig. 1. Thermal penetration depth around a fiber of stack; it is an effective distance for heat transfer between sound field and fiber. 
In both cases, for the efficiency and the coefficient of performance, the performance is lower than in a Carnot cycle, which is the term modified by $\Gamma$ in the above equation. Eqs. (1) and (2) are approximate since the viscous losses were omitted. Both equations show the intimate connection between heat and sound in thermoacoustics and the importance of geometric factors in the performance of such engines. The presence of a stack provides heat exchange with the sound field and the generation or absorption of acoustic power. With a suitable geometry substantial amounts of heat can be moved as demonstrated, for example, by Garrett and Hofler [5]. An interesting and important feature of such engines is that the performance depends on geometric factors and gas parameters, but it is independent of the device temperature [4]. Acoustic engines presented here were developed and scaled down so that they can be used for thermal management in circuits. Scaling the devices in size is based on principles of similitude, when possible [13], and on the development of components which are also reduced in size. The extension of the operating frequency range by over an order of magnitude requires special consideration of geometric factors, the dynamics of the devices, and material properties-thermal and acoustic.

It is interesting to note that the home refrigerator which uses a Rankine cycle based on a liquid-gas phase change has a coefficient of performance in the range of 2-7, depending on operating conditions. Hence, the performance of an acoustic cooler with comparable C.O.P. can lead to many applications.

This work presents a small acoustic cooler for device spot cooling and a prime mover for heat removal and even energy conversion.

\section{Interface with microcircuits}

\subsection{Acoustic cooler}

As part of the development of thermoacoustic engines for microcircuit heat transfer the present work discusses thermoacoustic refrigerators operated at frequencies in the range of 4-24 kHz. Typically an acoustic cooler pumped acoustically at $4.5 \mathrm{kHz}$ using a half-wave cylindrical resonator has a length of $\sim 4.0 \mathrm{~cm}$. It is driven by a piezoelectric driver, of the bimorph type, loaded with a cone; the stack of length $\Delta x=3-4 \mathrm{~mm}$ consists of random material such as cotton wool or glass wool evenly dispersed and it is in thermal contact with copper mesh heat exchangers at each end. The working gas is air at 1 atmosphere chosen because of the convenience of working with a simple fluid. Temperatures and temperature differences are measured by copper-constantan thermocouples connected directly to the heat exchanger at each side of the stack. The driver is powered by an audio amplifier activated by a signal generator. It was adjusted to be in tune with the resonator using a small sensing electret microphone. In order to minimize acoustic losses, the unit was fabricated to be acoustically leak-tight. This type of device can be used as a spot cooler on a circuit. Fig. 2 shows its interfacing to an element of the circuit, perhaps a transistor, which needs to be cooled below room temperature. The driver produces a standing wave in the resonator; heat is pumped acoustically from the cold heat exchanger toward the hot heat exchanger, away from the nearest pressure node. The hot exchanger dissipates the heat to the ambient by convection-cooling with fins attached to the hot heat exchanger unit, made out of copper.

The experimental set-up for testing the unit is shown in Fig. 3. A sine wave signal produced by a Stanford Research generator is amplified and sent to the driver; it is maintained at the resonant frequency of the cooler. The temperature of the hot and the cold heat exchangers is monitored separately with a copper-constantan thermocouple read by a Keithley multimeter, model 197A. Temperature measurement accuracy is $0.03{ }^{\circ} \mathrm{C}$ and the main source of error is from fluctuations and drifts of the reference couple anchored at the room temperature heat sink of the refrigerator. This error was comparable to the accuracy of temperature measurement. Resonant drive is maintained by monitoring the acoustic frequency with a miniature microphone coupled to the resonator. The sound level is monitored by a piezoresistive pressure transducer (National Semiconductor

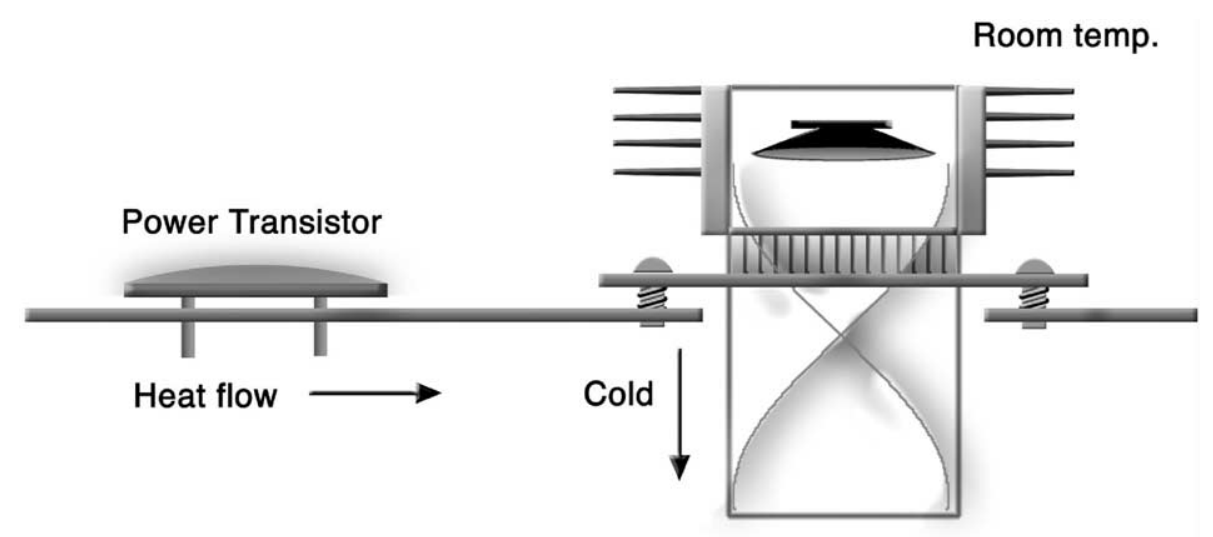

Fig. 2. Acoustic spot-cooler interfaced with circuit; heat from circuit is pumped to hot heat exchanger anchored thermally at room temperature. 


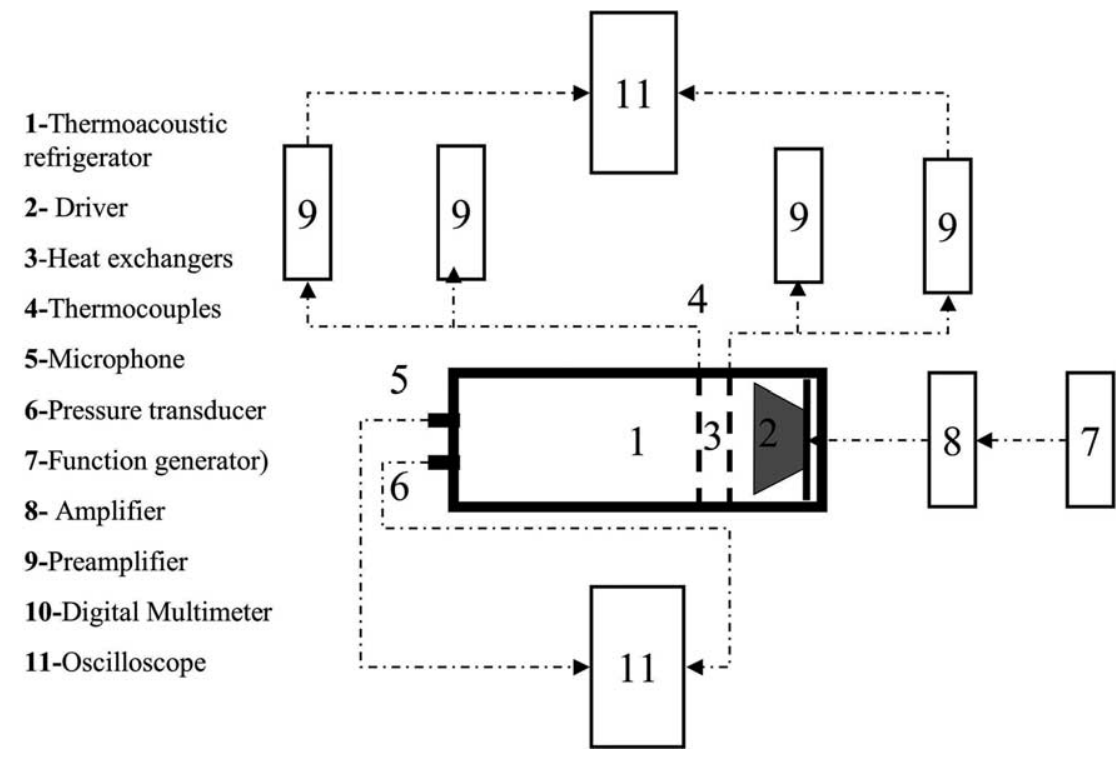

Fig. 3. Experimental set-up for acoustic cooler.

sensor LX1703AFN) also coupled to the resonator. The performance of the cooler is followed on an oscilloscope Tektronix TDS3012 with temperature on the $y$-axis and time on the $x$-axis.

A copper gasket is used for directly interfacing the cold heat exchanger of the cooler to the device which is to be cooled on the circuit. This provides simple access to the cold part of the cooler. One advantage of this type of interfacing is that the resonator and the working gas do not need to be cooled, thus reducing the thermal load on the refrigerator. Tests on a $4.5 \mathrm{kHz}$ refrigerator with both heat exchangers thermally floating are shown in Fig. 4. When the driver is activated on resonance at driver electrical power of $\sim 0.5$ watts the cold heat exchanger drops rapidly in temperature cooling by $4{ }^{\circ} \mathrm{C}$ in $50 \mathrm{~s}$. The hot heat exchanger with a substantially higher thermal load warms up by $7^{\circ} \mathrm{C}$. This creates a temperature difference $\Delta T$ of $11^{\circ} \mathrm{C}$ between the two heat exchangers. By thermally anchoring the hot heat exchanger to ambient temperature lower temperatures are reached by the cold exchanger. This is presented in Fig. 5, where the temperature of the cold heat exchanger is shown as a function of time when acoustic sound power is varied by means of the electrical power input to the driver. A cooling power of 1 watt can be achieved with a coefficient of performance of $2-3$. The performance depends strongly on geometry, power to the driver, and the quality of the driver. The efficiency of the driver by itself in converting electrical input into acoustic output is $\sim 35 \%$ at low electrical signals. When driven beyond its specifications the driver produces excessive heating which needs to be dissipated by the hot exchanger. The losses are caused mainly by hysteresis of the piezoelectric material and electrical contact resistance. The heat produced in the driver and the heat from the hot exchanger are dissipated by convection cooling in the fins attached to the hot copper chamber surrounding the driver and anchored thermally to the hot exchanger, as shown in Fig. 2. Multiple copper leads were used to conduct the heat from the driver to the copper chamber. The driver is rated for 25 volts continuous operation (Motorola bimorph, 1165). Further miniaturization of this type of refrigerator has led to operation in the ultrasonic range at $24 \mathrm{kHz}$, its length at that frequency being $0.8 \mathrm{~cm}$. Here as well the piezo is loaded resonantly by a cone.

The expected benefits of spot cooling electronic components affect significantly their electrical performance, heat transfer, and noise level. A recent evaluation of the advantages of circuit and device cooling in computers is presented in ref. [14].

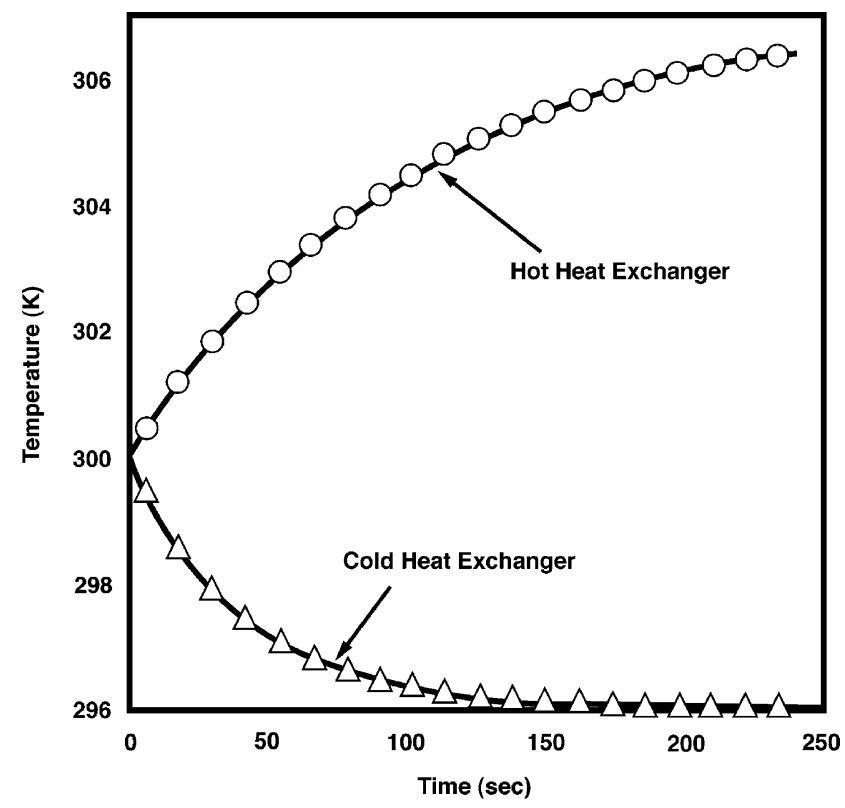

Fig. 4. Performance of an acoustic cooler with both heat exchangers thermally floating; acoustic power is kept constant. 


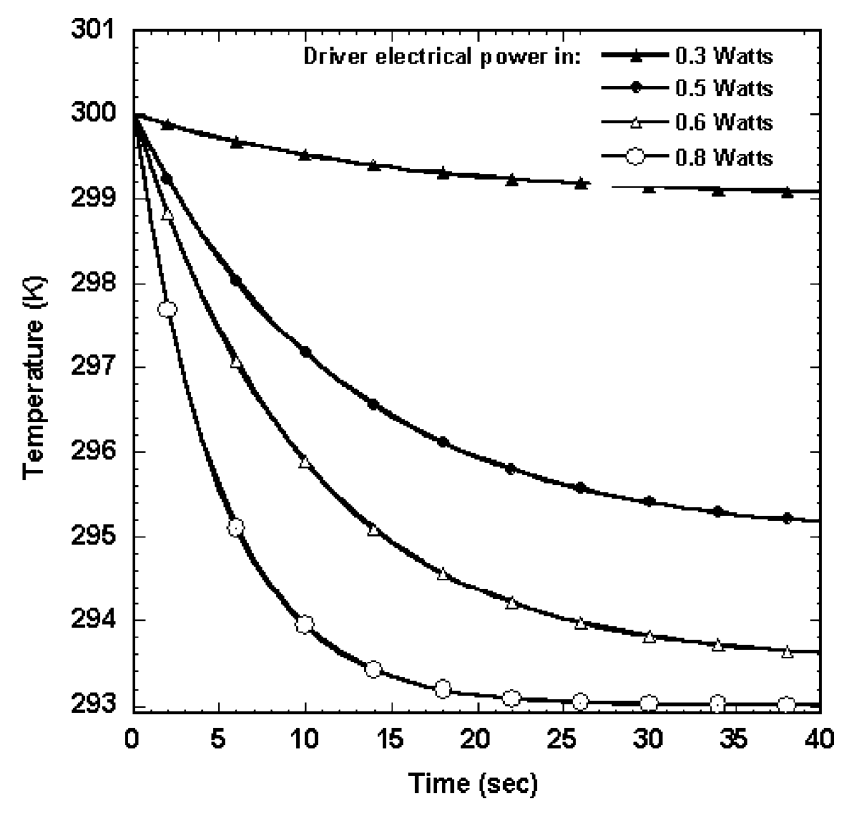

Fig. 5. Temperature change of cold heat exchanger when acoustic power is switched on at different electrical powers to the driver.

\subsection{Prime mover}

A prime mover can also be used to remove heat from a circuit by acoustic radiation, a simple and convenient application of the fact that heat above a threshold level and in a suitable geometry produces sound. In this case we use basically a $1 / 4$ wave resonator, typically $2 \mathrm{~cm}$ long. The hot heat exchanger is thermally anchored to the circuit while the cold exchanger is at room temperature. Due to heat flow from the circuit, a temperature difference $\Delta T$ across the stack is established; above the onset level for oscillations it causes acoustic oscillations. Thus heat is converted to sound. This is shown in Fig. 6. With a heat input of 2 watts and a $\Delta T$ of $40{ }^{\circ} \mathrm{C}$ across the stack, sound of $\sim 2 \mathrm{kHz}$ is produced at an intensity of $10 \mathrm{~mW} / \mathrm{cm}^{2}$. Such performance of this heat engine corresponds to $\sim 10 \%$ of Carnot efficiency. Similar devices of slightly different geometries and much larger size have demonstrated efficiencies in the range of $10-16 \%$ of Carnot [15]. Important features of this type of heat pump are the absence of moving parts, simple design, and small dimensions. The possibly annoying feature of the sound can be overcome or reduced in various ways: operation in the ultrasonic range, or in-situ immediate sound power conversion to electrical power. Further improvements of this type of engine for microcircuit applications can be made by lowering the threshold $\Delta T$ for oscillations (already this has been reduced down to $25^{\circ} \mathrm{C}$ ) and by incorporating the device into a parallel array. We have demonstrated this on a prototype with arrays of up to 18 parallel prime mover units. The advantages of an array are in the higher cooling power density (watts per unit volume) as a result of smaller units and in the shorter response time of the whole assembly. Interesting questions of phase-locking and coherence arise with an impact on power conversion. Arrays and in particular individual
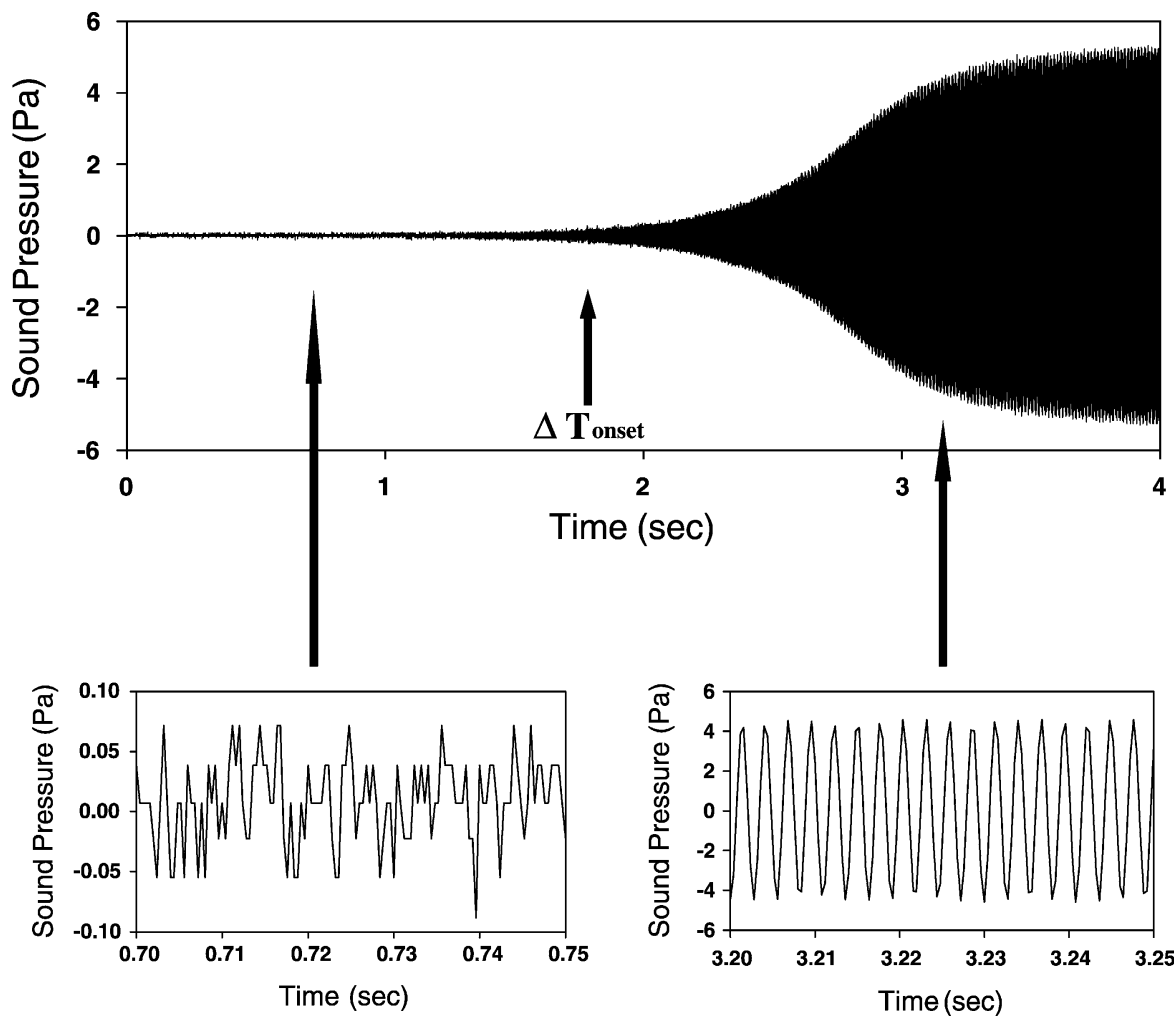

Fig. 6. Acoustic oscillations in prime mover above $\Delta T_{\text {onset }}$ applied across stack; heat flow to hot heat exchanger sustains the oscillations. 


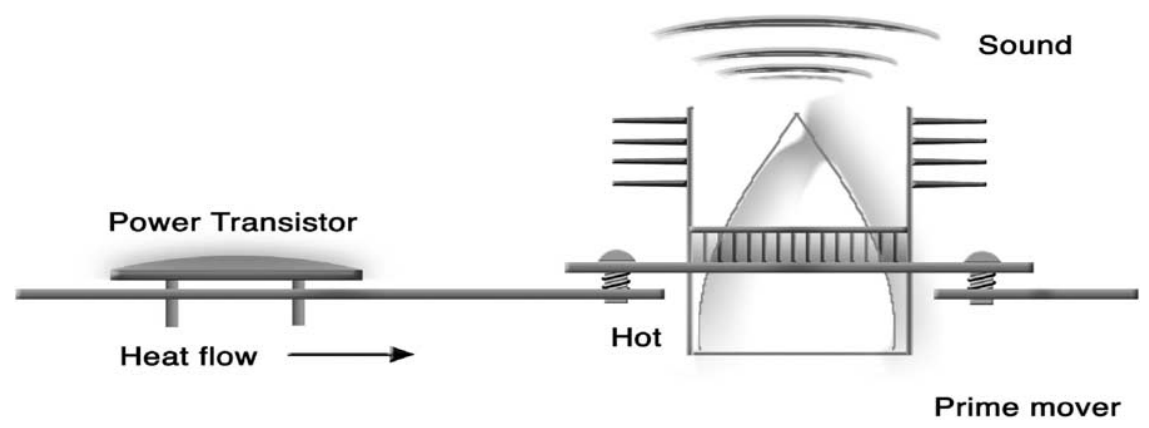

Fig. 7. Prime mover interfaced with circuit; heat is converted to sound.

small units operating in the ultrasonic range could be interfaced to microcircuit devices; they can be fabricated using microcircuit technology.

Fig. 7 shows the onset for oscillations of the prime mover as the temperature difference $\Delta T$ across the stack is increased; at the onset point random heat-generated pressure fluctuations, in the presence of a temperature gradient, trigger acoustic oscillations which start to become coherent and at the onset heat is converted to sound of essentially one frequency [16]. Larger temperature differences lead to a non-linear regime of oscillations followed by saturation in the level of acoustic oscillations. Fig. 7 shows the sound level as a function of time as the hot exchanger is being continuously heated while the cold heat exchanger is anchored thermally to room temperature, thus raising the temperature difference $\Delta T$ across the heat exchanger. Analysis of a larger prime mover below the onset for oscillation has been discussed by Atchley [17]. The approach taken here for heat transfer from a circuit can be compared to heat conduction along a solid conductor such as copper. In both cases a temperature gradient $\nabla T$ drives the heat flux across an element of cross-sectional area A:

$\dot{Q}=-K A \nabla T$

with $K=$ thermal conductivity of solid conductor. In the acoustic case $K=$ effective thermal conductivity of prime mover when producing sound [10].

A prime mover similar to the one described here has been reduced in size to the point that it radiates sound at frequencies as high as $20.4 \mathrm{kHz}$; this proves that this device can work in the ultrasonic range opening the way to further miniaturization.

The prime mover can also be used as the acoustic driver in the acoustic cooler. Although in that case the acoustic efficiency, determined by Eq. (3), is not high, the combination provides a simple unit with no moving parts which can be operated with low maintenance for long periods of time. Such approach demonstrated on a large scale [4] needs further development in the higher frequency ranges discussed here.

\section{Conclusion}

Two novel approaches have been presented using acoustics for managing and removing heat from microcircuits. They have much merit for such applications, especially from the point of view of simplicity, performance, environmentally safe components, and ease of interfacing with microcircuits. The acoustic approach compared to the standard passive copper/fins/convection approach provides substantial improvements for microcircuit applications in terms of efficiency, cost, and performance. Eq. (1) shows that high heat pumping rates can be achieved at small temperature differences. Our active device, the acoustic cooler, can provide spot-cooling below ambient; this is important for some applications. The unit that needs to be cooled, such as a chip, can be thermally anchored to the cold heat exchanger. A cooling power of 1 watt with a $\Delta T$ of $\sim 10{ }^{\circ} \mathrm{C}$ or more can provide substantial improvements in the speed and electronic device performance. By raising the operating frequency, the device becomes smaller and its response time is reduced. In a typical acoustic cooler, operating at $4.5 \mathrm{kHz}$, we have achieved cooling power densities of 0.1 to $0.6 \mathrm{~W} / \mathrm{cm}^{2}$. This is modest but encouraging since the devices can be optimized for higher performance by one to two orders of magnitude. Larger cooling power densities can be achieved by modifying the geometry (such as larger quality factor $\mathrm{Q}$ of resonator), raising the gas pressure [4], and using gas mixtures of lower Prandtl number [18]. Heat removal by acoustic radiation from a prime mover provides an approach, which works especially well over short distances from the heat source. An onset temperature difference of $25^{\circ} \mathrm{C}$ presented here is a very large improvement over onset temperature differences in previous low frequency prime movers; with further development this onset can be lowered to the $10{ }^{\circ} \mathrm{C}$ temperature difference range. Lowering this onset $\Delta T$ prevents the circuit from sustaining too high a temperature rise which could damage the components. This method of heat removal leads to substantial saving in the amount of heat conducting material that would have been needed in a passive approach. By raising our operating frequency, we have made the acoustic devices smaller so 
that they can be interfaced with microcircuits. The very high frequency range (the ultrasonic) provides an opportunity to develop arrays of microscale acoustic devices with high power densities. The results presented here point to further new developments by using the acoustic devices in array configurations. Although the demonstration of the performance presented here is preliminary, further developments will lead to major improvements. Miniaturized thermoacoustic devices can be interfaced individually with the chips on a microcircuit and they can be stacked in an array to service the whole unit, be it a desktop or a portable system. In such small geometries microfluid mechanics will be affected and they will have an interesting impact on the performance of the acoustic devices [19].

\section{Acknowledgements}

This research was supported by the Office of Naval Research, DARPA/SPAWAR, and the State of Utah.

\section{References}

[1] Y. Joshi, S. Garimella (Eds.), Thermal Challenges in Next Generation Electronic Systems, Millpress, Rotterdam, 2002.

[2] E. Abdel-Rahman, R. Behunin, Y. Kwon, O.G. Symko, Heat Transfer by High-Frequency Thermoacoustic Engines, in: J.P. Meyer (Ed.), Proceedings of the Second International Conference on Heat Transfer, Fluid Mechanics, and Thermodynamics, HEFAT, 2003.

[3] C. Tsai, R. Chen, C. Chen, J. DeNatale, Micromachined Stack Component for Miniature Thermoacoustic Refrigerator, MEMS 2002,
Fifteenth IEEE International Conference on Micro Electro Mechanical Systems, Piscataway, NJ (2002) p. 149.

[4] G.W. Swift, Thermoacoustic engines and refrigerators, Physics Today July (1995) 22.

[5] S. Garrett, T. Hofler, Thermoacoustic Refrigeration, Technology 2001. NASA Conference Publication 3136, vol. 2, 1991, p. 397.

[6] Lord Rayleigh (J.W. Strutt), The Theory of Sound, vol. 2, Dover, New York, 1945, Section 322

[7] P. Merkli, H. Thomann, Thermoacoustic effects in a resonance tube, Journal of Fluid Mechanics 70 (Part 1) (1975) 161.

[8] N. Rott, Thermally driven acoustic oscillations, Part III, Zeitschrift Angewandt: Mathamatical Physics 26 (1975) 43.

[9] N. Rott, G. Zouzoulas, Thermally driven acoustic oscillations, Part IV, Zeitschrift Angewandt: Mathamatical Physics 27 (1976) 197.

[10] N. Rott, Thermoacoustics, Advances in Applied Mechanics 20 (1980) 135 .

[11] G.W. Swift, Thermoacoustic engines, Journal of Acoustical Society of America 84 (1988) 1145.

[12] J.C. Wheatley, T. Hofler, G.W. Swift, A. Migliori, Experiments with an intrinsically irreversible acoustic heat engine, Physical Review Letters 50 (1983) 499.

[13] J.R. Olson, G.W. Swift, Similitude in thermoacoustics, Journal of Acoustical Society of America 95 (1994) 1405.

[14] M.J. Ellsworth Jr., The challenges of operating computers at ultra-low temperatures, Electronics Cooling 7 (3) (2001) 34.

[15] G.W. Swift, Analysis and performance of a large thermoacoustic engine, Journal of Acoustical Society of America 92 (1992) 1551.

[16] B. Chu, Analysis of a self-sustained thermally driven nonlinear vibration, The Physics of Fluids 6 (1963) 1638.

[17] A. Atchley, Standing wave analysis of a thermoacoustic prime mover below onset of self-oscillation, Journal of Acoustical Society of America 92 (1992) 2907.

[18] F.W. Giacobbe, Estimation of Prandtl numbers in binary mixtures of helium and other noble gases, Journal of Acoustic Society of America 96 (1994) 3568.

[19] N. Giordano, J.-T. Cheng, Microfluid mechanics: progress and opportunities, Journal of Physics: Condensed Matter 13 (2001) 12271. 